\title{
CONTROL AND OPTIMIZATION AGAINST EARTHQUAKE BY USING GENETIC ALGORITHM
}

\author{
K. Tajziehchi a ${ }^{\text {, A. Ghabussi }}{ }^{\text {b*}}$, H. Alizadeh ${ }^{\mathrm{c}}$ \\ ${ }^{a}$ Department of Civil Engineering, Islamic Azad University Central Tehran Branch, Tehran, Iran, e-mail: Khashayar.tj@gmail.com \\ b* Department of Civil Engineering, Islamic Azad University Central Tehran Branch, Tehran, Iran, e-mail: Ghabussi@outlook.com \\ ${ }^{c}$ Department of Civil Engineering, Islamic Azad University Central Tehran Branch, Tehran, Iran, e-mail: Hoseina01hah@gmail.com
}

Received: 07.01.2018 / Accepted: 13.02.2018 / Revised: 18.05.2018 / Available online: 31.05.2018

DOI: 10.2478/jaes-2018-0010

KEY WORDS: Time history analysis, Response spectrum, Plan spectrum, Genetic Algorithm, Hybrid Algorithm

\begin{abstract}
:
In this paper, controlling and optimizing against the earthquake by using genetic algorithm is investigated. In this paper, a new approach for selecting optimal accelerograph and scaling them for dynamic time history analysis is presented by the binary genetic algorithm and natural numbers, in order to achieve the mean response spectrum, which has a proper matching and a short distance with the target spectrum and indicates the expected earthquake of the site. Because of the difference in the nature of accelerograph and the scale coefficients, the genetic algorithm presented in this paper, is hybrid (has two chromosomes). The proposed algorithm is capable of constructing a new generation of people from a series of infinitesimal earth movement records, in a process where natural selection, mating, mutation takes place, and creates a new generation of people and continues this process until a person with desirable qualities is obtained. One of the most important factors in the accuracy and efficiency of these programs is the correct estimation of their parameters. If these parameters are correctly calculated, the difference between the mean response spectrum and the spectrum of the plot will be greatly reduced. Due to the relatively large number of these parameters, the use of trial and errorbased methods largely relies on user skills, the proposed hybrid genetic algorithm program can overcome this defect. The program has two genomes that run simultaneously and provide close answers to the optimal answer. The program itself is able to provide the user with a range of optimal coefficients and crossing values and mutations of each chromosome.
\end{abstract}

\section{INTRODUCTION}

Movement of the earth's motion and its durability are influenced by a variety of factors in one location, most notably the magnitude of the earthquake, the distance from the energy source, the condition of the soil, changes in the geological conditions and the velocity of waves' propagation in the path, the condition and mechanism of the earthquake source (type of fault, stress conditions and stress loss), according to records registered from previous earthquakes, it can be used to study the effect of these factors on the earth's motion. Kate Sanos et al. published an article entitled "Choosing earth's movements earthquake records: A New Idea from a Structural Engineering Viewpoint" in 2009. In this paper, the proposed method of selection is investigated based on the methods used to combine strong earthquake records in the framework of seismic design the structure. Due to the fact that certain records for a location are composed of a random process that is practically impossible to re-provide, in recent years a considerable effort has been made in processing actual records, so to become a representative of the existing input records and also construction in seismic areas in the future, planning has been done (Katsanos et al, 2010). In 2010, Irolino et al. proposed "Computer Assisted Record Selection for Structural Analysis by Seismic Regulations for Seismic Territories" (Iervolino et al,
2008). In 2011, Zhy Yang Ji et al. discussed a linear fragmentation of nonlinear issues. They subdivided the complex nonlinear problems into a linear objective function with a genetic algorithm and provided an approximation for solving the problem. Advantages of using the geometric algorithm in their research were the balance between the accuracy of approximation and simplicity and the improvement of approximations (Zhigang et al, 2011).

In this study, the control and optimization against earthquake using genetic algorithm is investigated. According to the literature, it can be seen that despite the consideration of Nanofluid displacement heat transfer in this type of compartment, Nano-fluid displacement heat transfer with the presence of a baffle has not been investigated. Assessment and identification of earthquakes that may occur in the future are important issues of earthquake engineering and structures, which requires the knowledge and prediction of the possible earthquake and its characteristics in the region, as well as the recognition of the structure behavior under this earthquake. In dynamic analysis methods, the earthquake side force is obtained by using the dynamic reflection of the structure caused by earthquake movement. These methods include the "spectral analysis" method and the "time history analysis" method. Earth motion, 
which is used in dynamic analysis, must have at least the earthquake condition of the plan. The effects of ground motion are determined in either of the two "acceleration reflection spectra" or "accelerated time histories". For the acceleration reflection spectrum, a standard design spectrum or a spectrum of site design can be used in accordance with the rules of the regulations. Generally, structures come into the nonlinear range when subjected to strong earthquakes, which is why the nonlinear analysis of the time history of the structure is important. Nonlinear analysis of time history is more common in seismic analysis and structural design. Seismic Separation Segregating Regulations include regulations governing nonlinear analysis of time histories. For nearly two decades, the rules of the regulations governing time histories have been described in Europe and the United States. Despite the fact that a seismic hazard in a site is provided for design purposes by the design spectrum, almost all design regulations, require a more precise method, to scale up and select the time history of the earthquake in accordance with the design spectrum.

\section{STATEMENT OF THE PROBLEM}

Regarding the main objective of obtaining the Earth's motion set by genetic algorithm, according to the Iran's 2800 regulation scheme, the genetic algorithm can be selected from among the actual earth mapping community for a specific area with a specific soil type, choosing and scaling to obtain a proper combination of erath's motion, in accordance with the design spectrum introduced in the Iran's 2800 regulation scheme. The research process is that, based on the characteristics of various earthquakes occurring in the world based on the type of soil, and the station's distance from the specified earthquake source, a database is selected, then these data are expanded for different types of soils and then the genetic algorithm will select the spectrum of the combination of these records by comparing the range of regulations, and if the convergence criterion is satisfied, that set is selected as an individual for the earthquake record (The response spectrum is derived from the expansion of sets that are proportional to the range of the rules of the regulation). The results show that the genetic algorithm produces accurate results in selecting and scaling the main earthquake sets in accordance with the design spectrum. The results can be used in design and consulting offices when dynamic analysis is required. Given the hypotheses such as comparing the spectrum of response with the design spectrum according to Iran's 2800 standard, the linearity behavior of the structures in the preparation of the response spectrum and the common assumptions for optimizing based on the Genetic Algorithm (GA) will answer such questions as the below questions. How to choose a suitable record compatible with the design spectrum for dynamic analysis? Do different parameters in the design of the genetic algorithm affect the results?

\section{APPLICATIONS OF GENETIC ALGORITHMS}

-Solve difficult issues,

-Some artistic uses include audio, video and multimedia combinations,

-Analysis and optimization of nonlinear dynamical systems, -Design and optimization of neural networks and the combination of neural networks and genetic algorithms in solving and optimizing certain issues (Sorkhabi, 2015).

\section{TIME HISTORIES ANALYSIS}

The earthquake-induced loads for different sites are usually expressed in terms of the proposed spectrum of seismic loading regulations. If the behavior of the structure remains within the linear range, the spectral analysis method can obtain the maximum values of the seismic response of the structure. However, these regulations require to use linear and nonlinear time histories analysis in a seismic analysis of irregular structures such as power plants, dams, high buildings, and structures with unconventional earthquake resistance, such as energy depreciating devices. Regardless the reasons of the need for dynamic time-zone analysis, input quakes in these analyzes should be presented as an accelerograph of the earthquake. But since seismic risk is expressed in a site in terms of the spectrum of the layout, the accelerograph values used in analyzing the history of time should be consistent with this spectrum (Haydar Kayhan et al, 2011). One of the most suitable methods for estimating the seismic behavior of buildings is the use of nonlinear analysis of time histories. For this purpose, earthquake records should be considered scale-wise. The Historical Time Method is introduced as a more precise method of spectral and static analysis in seismic design regulations, including the 2800 standard. For cases such as quantitative vulnerability assessment, the application of this method is essential for detailed, dynamically and sometimes non-linear analysis of the structure using historical stimulation charts. However, the application of this method to analysis and especially design in many practical cases faces uncertainties in determining the loading or identification of the site, which prevents the decision-making from responses resulting from a specific accelerograph even for the past earthquake. To carry out time history analysis, some accelerographs are needed that indicate the expected earthquake of the site. In most regulations, scaling of selected accelerographs is required to ensure that the spectrum of the response matches or exceeds the target range within a given time period.

The long duration of strong earthquakes returns over the period of accelerographs and seismic devices, as well as the possible nature of earthquakes, prevents it from being able to predict future movements of an area, even on a number of existing maps. In other words, for the design, it is better to use the range of regulations that, on the one hand, have been based on statistical studies on the large number of earthquake mappings and, on the other hand, has a legal right to design. The application of the standardized design spectrum is not directly applicable to dynamic analysis of time histories because accelerograph is the basis of analysis. In order to be able to use the features of existing earthquake mapping and target standard range. The most commonly used methodology, including standard 2800 , is to scale a specific number ( 3 or 7 pairs) of accelerographs, so that the spectrum obtained from them at a certain frequency range is not lower than the standard range. This method of regulation is equivalent to the application of the same coefficients of scale for different mappings, which can lead to a significant dispersion of the spectral values of the target and subsequently responses and is undesirable. In the last few decades, the first regulation 519 and then Iran's 2800 standard have been considered as the only official reference country for the seismic loading and analysis of structures and have been used in parallel with other international regulations. According to the regulations for designing buildings against 
earthquake, Iran's 2800 standard, designed to determine the minimum rules and regulations for seismic analysis of structures in order to maintain the stagnation of the structure against severe earthquakes and preventing losses, was developed and analyzed. Structural Model analysis is performed using two equally dynamic and dynamic static principal methods.

Dynamic analysis method, in turn, can be done in two ways: spectral analysis method and Dynamic Time Analysis method. Also in this regulation, criteria such as the height of the structure, regularity, etc. are used to determine the type of analysis method. A remarkable point in the proposed criteria is that, in some cases, spectral analysis and time histories are necessary to check and compare the results. Of course, in each case, the results should be compared with the results of the equivalent static analysis. The Earth's motion for dynamic analysis should also be determined by the accelerograph reflection spectrum or the history of accelerograph's variations. For this purpose, it is necessary to consider the appropriate earthquake records or the reflection range of its accelerograph, called the spectrum of the plot. The accelerographs used for the earthquake design should, as far as possible, reflect the actual movement of the earth from the earthquake site. In order to compare the results of dynamic analysis of time histories with equivalent spectral or static analysis methods, it is necessary to scale up the desired earthquake records. Different methods are presented for scaling the records. One of the methods, due to the relationship between the main rotational times, is the behavior coefficient, the coefficient of ductility and PGA, which results in a large dispersion in the results. Another method of scaling is the use of accelerograph parameters such as PGA in ground scaling, which creates a large dispersion in nonlinear accelerograph response spectra in long periods. Another method of scaling is based on the maximum ground motion features. The scattering of the results is remarkable and very large. Another method used in previous studies is to estimate the seismic response using spectral intensity, which results in a significant reduction in the distribution of the results when the spectral intensity of a series of earthquakes in the main period of the structure is taken.

\subsection{To scale up records}

In recent years, access to online data of the record for strong movements and real earthquakes has increased. Although according to the location of the record, the magnitude of the source earthquake, the type of failure, the type of soil, and, in general, the duration of the stroke, the distance between the source of the earthquake and the location of the ground motion record, the characteristics of the spectra can be very different. To this end, the specification of the seismic hazard of the site where the analyzed structure will be located is required to obtain or develop ground movements that are in accordance with a specific risk situation for the area defined by the implementation plan. In the new design regulations, the regional risk profile is described by the spectrum of the design and is generally described as a uniform hazard spectrum, in order to obtain an arbitrary range from the period of the structure. As no earthquake record has a response spectrum that is matched to the range of the rules of the code, there is no way to obtain consistent records based on the range of accelerograph that can be used in the design process (Haydar Kayhan et al, 2011). In order to be able to validate the history of time while observing the standard of the code, it is important to scale up accelerographs to comply with the scope of the draft regulatory framework. Regulations and guidelines provide different methods for scaling. Of course, most methods of scaling for linear dynamic analysis methods are presented. The main goal is to obtain an appropriate combination of a set with seven accelerograph land movements that is consistent with the spectrum of Iran's 2800 regulations. Therefore, the genetic algorithm based on the seismic region and soil type suggests the results for selecting and scaling actual earthquakes in order to obtain a set of inputs that are consistent with the range of the regulations. The program presented in this study is capable of navigating through a large database of accelerographs, which in this example includes 374 earthquake accelerographs, from Pierre's site with a shear wave speed of 360 to 750 that is suited to the type II soil with a very high seismic hazard of $0.35 \mathrm{~g}$ of 2800 standard, to select a set of 14 variables, 7 of which are earthquakes, and 7 of them are the scale coefficients of, in order to minimize the error of the response spectrum obtained from this set with the target range of the regulation by the squared square root equation. Over the past decade, elastic and nonelastic dynamical analyzes in the time domain have been made possible for complex structures with thousands of degrees of freedom, thanks to the increasing speed, computational power and evolution of software engineering. Therefore, time analysis is recommended in the vast majority of modern seismic regulations.

\subsection{The use of genetic algorithm to scale records}

The selection and scaling of earthquake records that are matched to the design spectrum can be formulated with engineering optimization issues such as squared squares, the distance between the response spectrum and the average response range selected and scaled within the desired period. The current methods of regulation that result in the same scale factor for an accelerograph set will not necessarily lead to the closest match of the average range resulting from the selected accelerographs with the standard design spectrum, but the achievement of such a goal should be in the form of optimization of the combination composition coefficients. Several algorithms, which can be categorized as definite or randomized solutions for engineering optimization problems, have been developed. Gradient-definite algorithms are local search methods that require significant gradient information to find solutions. Generally optimal finding using gradient algorithms is not easy unless the results space is clear. That's why the interest in the use of random optimization algorithms to solve engineering optimization problems has increased. Among the randomization algorithms used, the genetic algorithm and etc. can be used. The goal is to use the genetic algorithm to solve the problem. The error obtained from the objective function with the mean squared error is between the squared square root of the mean of the scaled spectrum and the target spectrum, so that the results of the average range scaled above the target range are in the range from T0 to Tn. The search process obtains the best seven seismic couples and their compatible scale coefficients. There is also no limitation in choosing the number of records and scaling coefficients that the algorithm may choose. 


\subsection{Basic elements of the genetic algorithm applied to these issues}

POPULATION: is a set of hypothesized solution variables. In most programs there are thousands of "person" in the crowd These individuals are binary strings that, after being decoded, are evaluated to true and integer numbers that show problem variables for "natural selection of parents." The primary population is usually provided randomly. The generations of children are propagated by the operators of the genetic algorithm (intersection and mutation) to parents in the population. In this research, the number of people in 50,100 and 300 , respectively, has been selected in separate programs and finally the amount of population suitable for this issue has been introduced.

Fitness function: This is a mathematical expression for assessing the fitness of individuals in generations. The main rule in defining a fitness function is that those who are close to optimal must have larger values than surrender. As a result, those who are more likely to receive a higher chance of being elected as a parent in the next generation (Pezeshk et al., 1999). Intersection: This process is carried out by the combination of two individuals for reproduction and birth. This is done by modifying and sharing parts of the parent's profile. Several patterns of intersections such as single-point intersection, multipoint, and intersection are uniform. In this study, a uniform intersection for the binary section of the genetic algorithm and the intersection of the mixture for the real segment of the genetic algorithm is used. The intersection rates for the three values were $0.6,0.65$ and 0.9 , respectively.

Mutations: is an essential mechanism for ensuring diversity in the population. When a person is randomly selected to be mutable (by enabling the possibility of a mutation), the algorithm randomly selects bits in the range 0 and 1 , or duplicates in the opposite direction to a constant pattern of solutions for reproduction among all future generations. It is necessary that a vast search of all search space be done. The probability of a very large mutation, however, can improve the process for adaptation and early convergence. In this case, the mutation rate has been investigated for three values of 0.001 , 0.01 and 0.025 .

Natural parent selection: This is a possible method of choosing based on individuals' fitness. To ensure the survival of the best, people with a higher fitness level have a higher chance of being elected as a parent for birth in the next generation. In this study, the type of selection is a race.

\subsection{Selecting accelerograph for seismic pattern}

Structural design cycles require earthquake loads to compare with a smooth response spectrum as inputs in dynamic analysis. Input moves should be chosen to represent the seismicity of the area. In other words, real records should provide details of how to conduct an earthquake in order to use in the context of transient dynamic analysis. In this research, a collection of 374 records of the modified accelerograph pairs has been extracted to the actual component of 24 earthquakes with 5\% damping from the Pierre site (University of Berkeley). The average shear wave velocity at the recording site of these accelerographs, is 360 to 750 , in accordance with standard 2800 Type 2 soil with a high relative seismic risk area of $0.35 \mathrm{~g}$. The genetic algorithm will select records from this set and compare the spectral response of this accelerograph pairs in the range of the period of time from 0.25 to 1.86 seconds with the dT interval of 0.1 . The choice and scale of earthquakes in accordance with the design spectrum set forth in the 2800 regulation is proposed as an optimization problem.

\subsection{Programs run at different stages and provide evolved program}

In this research, a code was first written for the target function and proposed reference, and it was implemented in the MATLAB the genetic tool software. The results of the study had at least three repetitive earthquakes and high error rates. One of the suggested solutions for the problem is using the correct component function, which itself causes a large rounding error in the optimization process. The author sees the cause of this early convergence in the inability of the MATLAB Software's genetic tool to work with integers. One of the other reasons that causes a lot of errors in this issue is the lack of attention to the fact that the study problem has two sets of variables (scale coefficients and earthquake numbers). Therefore, the author considers making the problem as twochromosome. In this regard, he has tried to combine the genetic numbers of real, binary, and permutable algorithms efficiently to reduce the error of the plot. In the first step, due to the high speed of the natural number genetic algorithm compared to the binary numbers, the problem was designed as a combination of natural numbers algorithm for scale coefficients and a permutation algorithm for earthquake numbers. This led to a dramatic reduction in the error rate, but according to the values of the objective function, it was deduced that the real numbers genetic algorithm with the crossing mean value reduces the search power of the plan and reduces the accuracy of the algorithm. In the sequel, the genetic algorithm of real numbers with directional and combinational crossing was used to increase the search power of the proposed algorithm, which caused the algorithm to diverge and provide points beyond the acceptable range as the optimized point. In order to increase the accuracy and reduce the design error of combination of binary and permutation algorithms, the results show a significant reduction in the design error, while there is no significant change in the time of obtaining, this suggests that the nature of the chromosome expressing scale coefficients is more consistent with the binary algorithm, then the results of both proposed algorithms are presented and examined.

\subsection{Presentation of Hybrid Genetic Algorithm (Selection of Optimization Control Parameters by Genetic Algorithm)}

Due to the close approximation of errors to each other, one of the most important factors in the accuracy and efficiency of these programs is the correct estimation of their parameters. If these parameters are correctly calculated, the difference between the spectrum of the response and the spectrum of the plan will be greatly reduced. Considering the relatively large number of these parameters, the use of trial and error-based methods largely relies on user skills, which is why this research is a hybrid genetic algorithm program written to enable beginner users who have no diagnostic power for favourable values of optimization parameters. The program itself is able to provide users with the range of optimal coefficients and crossing values and mutations of each chromosome. The hybrid genetic algorithm can compete with previous methods, which means 
that it can determine the values of optimization control parameters by the other genetic algorithm that is included in the first genetic algorithm and reach optimal and near optimal results in the shortest possible time and easily provide to the user. The algorithm presented in this section is shown in Fig. 1.

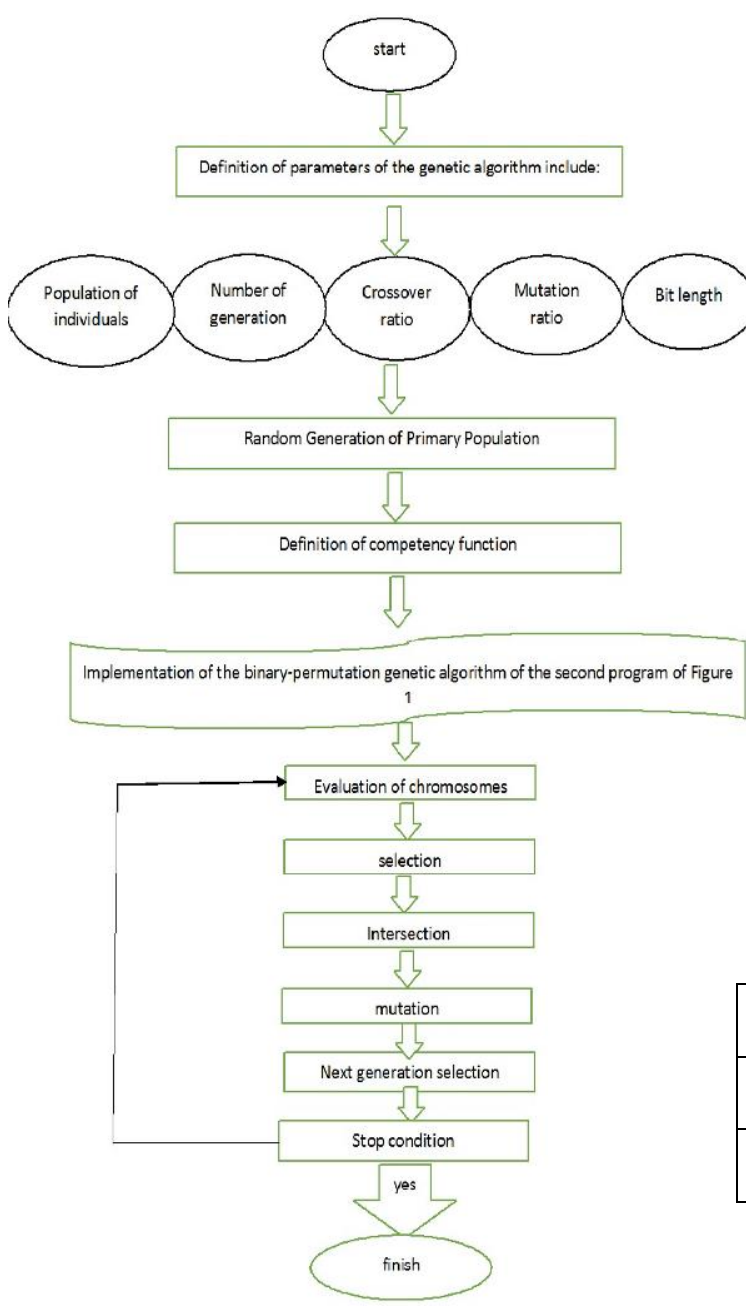

Figure 1. Hybrid Genetic Algorithm Structure

- the program implementation with the proposed values by the genetic algorithm for binary encryption

Finally, the program of hybrid genetic algorithms has been able to introduce the optimization control parameters. These values are presented by the Hybrid Genetic Program in Table 1.

Table 1. The values of the parameters obtained from the hybrid algorithm

\begin{tabular}{|c|c|c|c|c|c|c|}
\hline $\begin{array}{c}\text { Scaling } \\
\text { range }\end{array}$ & $\begin{array}{c}\text { Popula- } \\
\text { tion size }\end{array}$ & $\begin{array}{c}\text { Number } \\
\text { of } \\
\text { genera- } \\
\text { tions }\end{array}$ & $\begin{array}{c}\text { Cross- } \\
\text { ing rate } \\
\text { of chro- } \\
\text { mosome } \\
1\end{array}$ & $\begin{array}{c}\text { Cross- } \\
\text { ing rate } \\
\text { of chro- } \\
\text { mosome } \\
2\end{array}$ & $\begin{array}{c}\text { Muta- } \\
\text { tion rate } \\
\text { of chro- } \\
\text { mosome } \\
1\end{array}$ & $\begin{array}{c}\text { Mutat- } \\
\text { ion rate } \\
\text { of chro- } \\
\text { mosome } \\
2\end{array}$ \\
\hline $\begin{array}{c}3259- \\
1.0005\end{array}$ & 200 & 300 & 0.268 & 0.317 & 0.576 & 0.047 \\
\hline
\end{tabular}

After five times the implementation of the binary genetic program with the values of the hybrid algorithm, we arrived at the results of table 2 and 3 that is shown below.
Table 2. Results of program implementation with values obtained from hybrid algorithm

\begin{tabular}{|c|r|r|r|c|c|c|c|}
\hline $\begin{array}{c}\text { implementation } \\
\text { number }\end{array}$ & \multicolumn{7}{|c|}{ Scale coefficients } \\
\hline 1 & 0.62 & 0.47 & 0.5 & 0.7 & 0.42 & 0.4 & 0.6 \\
\hline 2 & 0.79 & 0.47 & 0.9 & 0.4 & 0.85 & 0.7 & 0.9 \\
\hline 3 & 0.79 & 0.68 & 0.6 & 0.7 & 0.43 & 0.4 & 0.4 \\
\hline 4 & 0.89 & 0.88 & 0.6 & 0.7 & 0.71 & 0.6 & 0.6 \\
\hline 5 & 0.84 & 0.7 & 0.9 & 0.5 & 0.47 & 0.6 & 0.9 \\
\hline
\end{tabular}

Table 3. Results of program implementation with values obtained from hybrid algorithm

\begin{tabular}{|r|r|r|r|r|r|r|r|c|}
\hline $\begin{array}{c}\text { implementation } \\
\text { number }\end{array}$ & \multicolumn{6}{|c|}{ Number of earthquakes } & Error \\
\hline 1 & 49 & 37 & 168 & 53 & 78 & 159 & 176 & 0.0313 \\
\hline 2 & 21 & 37 & 53 & 78 & 296 & 10 & 168 & 0.0352 \\
\hline 3 & 347 & 183 & 296 & 37 & 214 & 233 & 172 & 0.0292 \\
\hline 4 & 168 & 53 & 176 & 21 & 347 & 10 & 78 & 0.0171 \\
\hline 5 & 233 & 77 & 53 & 10 & 78 & 39 & 296 & 0.038 \\
\hline
\end{tabular}

The minimum error obtained by binary encoding with the proposed genetic values was $1.7 \%$. Table 3 shows the scale coefficients and earthquake numbers that were obtained from the fourth implementation of this program.

Table 4. Scale coefficients and number of earthquakes selected from the hybrid algorithm

\begin{tabular}{|r|r|r|r|r|r|r|r|}
\hline \multicolumn{7}{|c|}{ Minimum error obtained by hybrid algorithm = 1.71\% } \\
\hline $\begin{array}{r}\text { Scale } \\
\text { coefficients }\end{array}$ & 0.885099 & 0.875208 & 0.59363 & 0.748597 & 0.705733 & 0.564615 & 0.619348 \\
\hline $\begin{array}{r}\text { Number of } \\
\text { earthquakes }\end{array}$ & 168 & 53 & 176 & 21 & 347 & 10 & 78 \\
\hline
\end{tabular}

Figure 2 illustrates the process of improving the suitability function that the program implemented using the hybrid algorithm.

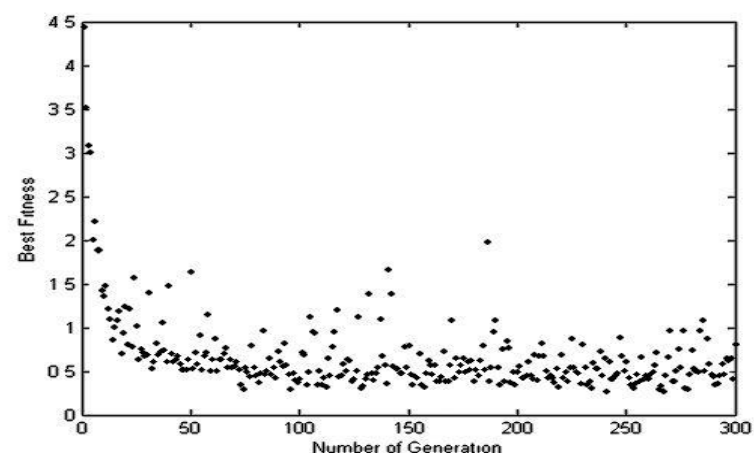

Figure 2. The fitness diagram of the hybrid program

Fig. 3 shows the medium response spectrum graph of total square root of best implementation (fourth implementation) of the hybrid algorithm program compared to Iran's 2800 regulations spectrum. 


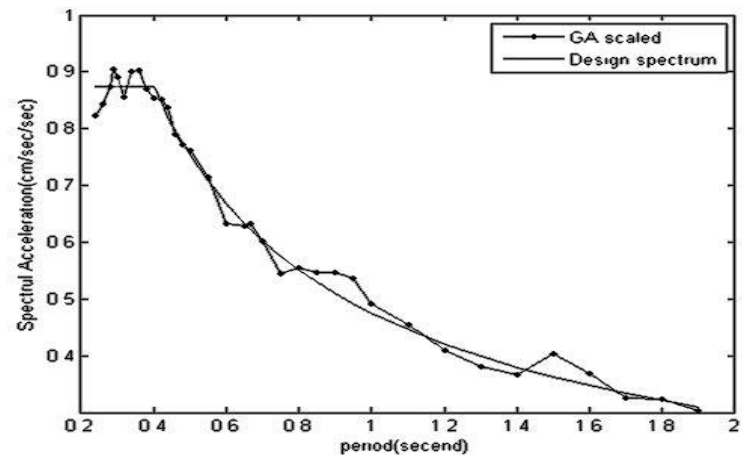

Figure 3. Comparison of the response spectrum obtained by the hybrid algorithm with the spectrum of the design

In Figure 4, a bar chart is drawn up for comparing and evaluating the results of the implemented programs in 22 different combinations.

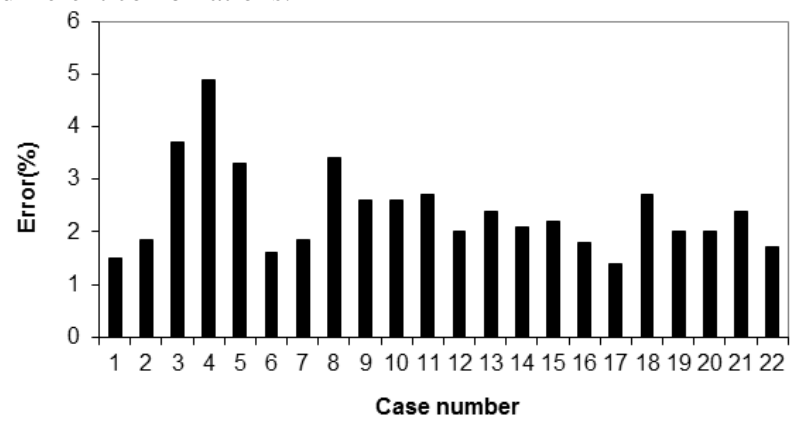

Figure 4. A bar chart to compare the errors of 22 combinations of two programs of binary and real genetic algorithms

\section{CONCLUSION}

In this paper, control and optimization of seismicity using genetic algorithm was investigated. The results show that:

1) In the case of an occurrence probability, the low combination leads to the selection of repetitive accelerographs.

2) The number of generations is not good if the number of people is low.

3) The number of population in real genetic algorithms ranges from 200 to 500 .

4) The mutation rate in the integer genetic program is more flexible than binary mode.

5) The higher the range of coefficients, the more dispersion is obtained in the answer.

6) The larger the database of accelerographs data, the larger the search algorithm's space, and the more favourable results are obtained.

\section{REFERENCES}

Katsanos, EI., Sextos, AG., Manolis GD., 2010, Selection of earthquake ground motion records: a state-of-the-art review from a structural engineering perspective, Soil Dynamics and Earthquake Engineering ,30:157-69.

Iervolino, I., Maddaloni, G., Cosenza, E., 2008, Eurocode 8 compliant real record sets for seismic analysis of structures, Journal of Earthquake Engineering, 12(1):54-90.

Zhigang, Ji. Zhenyu, Li., Zhiqiang, Ji. 2011, Research on genetic algorithm and data information based on combined framework for nonlinear function optimization.

Pezeshk, S., Camp, C. V., and Chen, D., 1999, Genetic algorithm for design of nonlinear framed structures, Proceedings of the 1999 Structures Congress, New Orleans, Louisiana, ASCE.

Shahrouzi, M., Sazjini, M., 2012, Refined harmony search for optimal scaling and selection of accelerograms, Scientia Iranica $\mathrm{A}(2012)$ 19(2), 218-224.

Haydar Kayhan, A., Armagan Korkmaz, K., Irfanoghlu, A., 2011, Selecting and scaling real ground motion records using harmony se arch algorithm.

Sorkhabi, O. M. (2015), Geoid Determination Based on Log Sigmoid Function of Artificial Neural Networks: (A case study: Iran), Journal of Artificial Intelligence in Electrical Engineering, Vol. 3, No. 12, pp. 18-24. 